\section{Re: Patient-Provider Communication: Does Electronic Messaging Reduce Incoming Telephone Calls?}

To the Editor: In "Patient-Provider Communication: Does Electronic Messaging Reduce Incoming Telephone Calls?"1 the authors concluded that in fact, electronic messaging between patients and providers is associated with increased phone calls, counterintuitive to the notion that electronic portals might help decrease overall workload. Although this is an important distinction to make, I will argue that patient satisfaction and doctor-patient communication are ultimately better reasons for on-line portal use than decreasing workload. We would all like to reduce administrative burden; however, studies show physicians value a manageable workload more than working fewer hours, ${ }^{2}$ and electronic portals offer many benefits to patients. ${ }^{3}$

Patient portals improve patient self management of chronic disease and improve quality of care, especially patient-provider communication. ${ }^{3}$ Access to electronic messaging between patients and doctors is significantly associated with improved clinical outcomes and enhanced patient satisfaction. ${ }^{4}$ Intuitively, patient satisfaction may lead to an increased personal accomplishment in physicians. Doctors with an increased sense of personal accomplishment are happier. ${ }^{2}$

Conventional wisdom states that doctors are unhappy because they are overworked. ${ }^{5}$ Physicians and other members of the health care team may be resistant to using patient portals if it is shown that they do not reduce workload. However, the number of hours worked is not related to physician happiness. Rather, workload manageability is significantly related to happiness. ${ }^{2}$ The concept of workload manageability is open to interpretation, but some options include increased support staff, flexible scheduling, and a greater voice in workplace matters. Future studies should explore this idea of a manageable workload, what it means to doctors, and how to best support us in this endeavor.

Haley Bittner, MD

Riverside Methodist Hospital Family Medicine

Residency Program

Columbus, $\mathrm{OH}$

haley.bittner@ohiohealth.com

To see this article online, please go to: http://jabfm.org/content/ 29/7/109.full.

\section{References}

1. Dexter E, Fields S, Rdesinski RE, Sachdeva B, Yamashita D, Marino M. Patient-provider communication: does electronic messaging reduce incoming telephone calls? J Am Board Fam Med 2016;29:613-9.

2. Eckleberry-Hunt J, Kirkpatrick H, Taku K, Hunt R, Vasappa R. Relation between physicians' work lives and happiness. South Med J 2016;109:207-12.

3. Goldzweig CL, Orshansky G, Paige NM, et al. Patient portals: Evidence on health outcomes, satisfaction, efficiency, and attitudes: A systematic review. Ann Intern Med 2013;159:677-87.

4. Wade-Vuturo AE, Mayberry LS, Osborn CY. Secure messaging and diabetes management: Experiences and perspectives of patient portal users. J Am Med Inform Assoc 20(3)13;20:519-25.

5. Smith R. Why are doctors so unhappy? There are probably many causes, some of them deep. BMJ 2001;322:1073-4.

doi: 10.3122/jabfm.2017.01.160324

The above letter was referred to the authors of the article in question, who offer the following reply.

\section{Response: Re: Patient-Provider Communication: Does Electronic Messaging Reduce Incoming Telephone Calls?}

To the Editor: We appreciate the thoughtful correspondence. Our article addresses the question of what would happen to phone call volume in the face of expanding electronic messaging. Although we offered multiple hypotheses about why we had unexpected findings, the reason for this outcome is unclear and deserves additional study. Dr. Bittner rightly points out that electronic communication with patients has many possible benefits. But the benefits of new health care interventions must be empirically tested and rigorously examined to best delineate their true value to care. In this case, we feel we have demonstrated that electronic portals have not yet been shown to be important in reducing other types of work, and clinics need to be aware of this. In addition, one must remember that the system of care that we design must be sustainable financially as well as emotionally. In a fee-for-service environment, clinicians are the driving force of reimbursement. This means that they may not have time to devote to portal communication if it takes them away from revenue-generating activities. In a capitation- or value-based model, there is the opportunity to build teams to serve patients in different ways. In this environment, financial resources may be available to support non-visit-based care, including both electronic and telephone communication by clinicians and staff. Our study demonstrated that there is not an obvious trade-off between electronic and telephone communication, and prac- 